\title{
Enhanced constrained local models for gender prediction
}

\author{
Ayah Alsarayreh, Fatma Susilawati Mohamad \\ Faculty of Informatics and Computing, Universiti Sultan Zainal Abidin Besut, Besut, Malaysia
}

\begin{tabular}{l} 
Article Info \\
\hline Article history: \\
Received Mar 5, 2021 \\
Revised Jun 14, 2021 \\
Accepted Dec 25, 2021 \\
\hline Keywords: \\
Constrained local models \\
Face landmark \\
Gender prediction
\end{tabular}

Gender prediction

\begin{abstract}
Face land-marking, defined as the detection and positioning of distinctive characteristics, is a crucial goal shared by various organizations, ranging from biometric recognition to mental state comprehension. Despite its apparent simplicity, this problem has been extensively investigated because of inherent face variability and a variety of confusing variables such as posture, voice, illumination, and occlusions. In this paper, an integrated mount model is created to increase the power of constrained local models, and a ground-breaking result for feature detection is obtained using this model. Furthermore, four classifiers have been used in the level of gender prediction. The results of the experiment showed that the proposed model performs admirably.
\end{abstract}

This is an open access article under the CC BY-SA license.

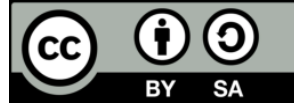

\section{Corresponding Author:}

Ayah Alsarayreh

Faculty of Informatics and Computing, Universiti Sultan Zainal Abidin

Besut, Campus, 22200 Besut Campus, 22200 Besut, Terengganu, Malaysia

Email: aya_izzat_89@yahoo.com

\section{INTRODUCTION}

The face feature is defined as the recognition of distinct facial characteristics. The points represent critical information required for classifying an individual; this is achieved by developing a model [1]. The model is composed of a certain number of landmark points, which is defined by the complexity of the object's shape and the level of detail necessary for its location. Facial landmarks are sometimes referred to as facial feature points, anchor points, homologous points, and essential points in the literature. Numerous techniques consider the image of the face and the collection of facial feature points to be a single shape [2]. These methods take advantage of prior knowledge about the position of the face (gleaned from labeled training images) and constrain the landmark searching using heuristic criteria such as areas, angles, and distances. As a result, the approaches can be used to estimate the shape of a previously unknown face [3]. These approaches include active shape modeling (ASM) [4], active appearance modeling (AAM) [5], and constrained local model (CLM) [6]. They evaluated the AAM and ASM models in [7] and discovered that ASM outperformed AAM in terms of speed and success in predicting the location of the feature point.

The AAM uses a model of the appearance of the entire facial region, Whereas ASM constrains to models the image texture nearest to each landmark point. ASM is quicker and has an extensive search domain than AAM, while AAM better identifies the texture [7]. AAMs can find a proper place for the head. Hence, give accurate pose estimation, and this considers as the advantage of AAMs. However, the accuracy of AAMs depends mainly on the used training set. So, to enable AAM to work on different faces, it should provide several faces with different poses in the training set, and this is the main limitation of AAM. CLM model depends on the express nearby facial appearance and expresses global facial shape pattern.

The regression-built strategies utilization holistic or nearby appearance majority of the data; furthermore, they might implant those global facial state patterns implicitly to joint landmark discovery. The 
CLM model has brought remarkable developments as far back as a few years. Zadeh et al. [8] Introduced as a local detector, the deep constrained local model (DCLM) method and the novel dense projection network (DPN). DPN will be a deep neural network consisting of two vital layers: a thick aggravator layer of projection format. For the format projection layer, patches from claiming facial area should be mapped for a higher dimensional space that allows the pose to be caught faultlessly by revolutionary varieties.

A set of masters replicated in a dense aggravator layer inside one network should exacerbate the assignment of an additional vital point of interest limitation. Zahraddeen et al. [9] develops a feature extraction strategy by combining the Gabor filter and the discrete cosine transform (DCT). The Gabor filter approach turned the images into Gabor magnitude and then smoothed them with an image smoother before applying the transformation. The effect of the Gabor filter on the DCT coefficients was evaluated using coefficient correlation. Geng et al. [10] developed deep learning algorithms for eliciting facial features based on convolutional neural networks $(\mathrm{CNN})$. In contrast to the previous models generated using $\mathrm{CNN}$, these used a trait map derived from separate layers. Numerous strategies have been applied recently to extract facial points or features from photos to improve feature extraction [11]-[13].

In [14], developed inadequate reconstruction technique to those face alignment issues. As opposed to a direct regression in the middle of those characteristics and shape space, the idea for shape augments reconstruction is presented. Moreover, a situated of coupled over complete studies termed those shape increase, and the local appearance is figured out in a regressive way on select deep characteristics. A constraind local model is considered as a class of method of locating set of points on the target image. Much researchs have been devoted to study the chractrisation of this model for instans see [15]-[20]. This article proposed an improved CLM. That gathers the whole quality of both depth and strength data to identify and monitor facial expressions in pictures. The depth data helps us to minimize the impact of lighting conditions. Moreover, it let us decrease the influence of the problem of aperture, which emerges on account of the strong patch response over the edges but not across them. On the other hand, our method uses depth reaction CLM only when there is no strong signal or inadequate lighting conditions.

Many different types of face recognition research have been conducted with the goal of gender prediction; for example, see [21]-[26]. This paper employs the A-CLM model for gender prediction; at this point, four classifiers are used to do this, suport vector machine (SVM), support vector regression (SVR), knearest neighbors (KNN), and progressive transductive SVM (PT-SVM). In this study, the most significant advance is adopting a constrained local model with three patch experts, which is a radical departure from the previous paper's constrained local model with a single patch expert.

\section{RESEARCH METHOD}

This section contains an explanation of the methodology and procedures that were employed in this study. The proposed technique is described in detail in section 1 . The second section covers the database that was used, and the third section illustrates the point distribution model (PDM). Section 4 discusses patch experts, while section 5 provides a summary of the fitting procedure.

The feature extraction technique was carried out in three stages in this article. As seen in Figure 1. Because our approach is based on the CLM structure, it is detailed here. As illustrated in Figure 1, CLM has three essential components: a PDM, patch expert, and fitting technique. The PDM model locates facial feature foci in the image by utilizing non-rigid shape and rigid global transformation factors. Using patch specialists, one may simulate the appearance of neighborhood patches surrounding significant monuments. The following subsections detail the steps in greater detail.

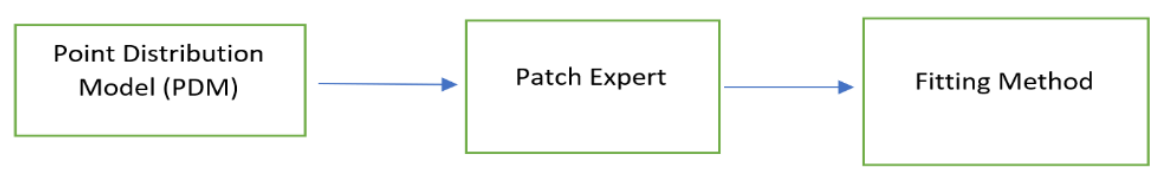

Figure 1. The enhanced CLM basic components

\subsection{Point distribution model}

Variables $p=[s, R, q, t]$ can be defined in our modified CLM model, and they can be diverted to obtain a specific model. In this equation, $s$ represents the scaling factor, $R$ represents object rotation, $t$ represents $2 D$ translation, and $q$ is a vector indicating non-rigid form variation. In PDM, the following setup is utilized in A-CLM: 


$$
x_{i}=s R_{2 D}\left(\bar{x}+\phi_{i} q\right)+t
$$

Here, $\bar{x}_{i}=\left[\overline{x_{l}}, \bar{y}_{l}, \bar{z}_{l}\right]^{T}$ denotes to the mean quality of the $i^{t h}$ characteristic as a vector, $\phi_{i}$ represent a matrix of size $3 \times m$ of principal component, where $q$ stand to the $m$ dimensional vector for variables monitoring the non-rigid shape. Rather than using perspective projection, this method makes use of a weakperspective (scaled orthographic) camera model, as linearity allows for more efficient optimization. The scaling factor s can be used in place of the average width in a weak-perspective model, and the translation vector $\mathrm{t}$ can be used as the center point. Due to the relatively minor fluctuations in depth along the face plane, this is a good approximation for the distance to the camera. We determine the maximum a posteriori probability (MAP) of the face model parameters $\mathrm{p}$, in the proposed model.

$$
p\left(P \mid\left(l_{i}=1\right)_{i=1}^{n}, I\right) \alpha p(P) \prod_{i=1}^{n} p\left(l_{i}=1 \mid x_{i}, I\right)
$$

Here, $l_{i} \in\{-1,1\}$ indicates whether the $\mathrm{i}^{\text {th }}$ characteristic point will be modified or skewed, $p(p)$ indicates the former likelihood of the model parameters $p$, and $\prod_{i=1}^{n} p\left(l_{i}=1 \mid x_{i}, I\right)$ indicates the joint likelihood of the characteristic focuses $x$ continuously adjusted at a specific point $x_{i}$, provided for a force intensity i. To calculate $p\left(l_{i}=1 \mid x_{i}, I\right)$, the patch experts are normally used, which is the likelihood that a function is aligned at $x_{i}$.

\subsection{Patch experts}

We determine how the new feature points are matched depending on the regional support area by using local patch experts who calculate the likelihood of alignment $p\left(l_{i}=1 \mid x_{i}, I\right)$. We use (3) as a probabilistic patch expert; the mean value of two logistic regression (4) and (5).

$$
\begin{aligned}
& p\left(l_{i} \mid x_{i}, I, Z\right)=\frac{1}{2} \times\left(p\left(l_{i} \mid x_{i}, I\right)+p\left(l_{i} \mid x_{i}, Z\right)\right) \\
& p\left(l_{i} \mid x_{i}, I\right)=\frac{1}{1+e^{d C_{I, i}\left(x_{i} ; I\right)+c}} \\
& p\left(l_{i} \mid x_{i}, Z\right)=\frac{1}{1+e^{d C_{Z, i}\left(x_{i} ; Z\right)+c}}
\end{aligned}
$$

Here $C_{Z, i}$ and $C_{I, i}$ are the outputs of the strength and depth patch classifiers, respectively $c$ is the logistic regressor intercept for the $i^{\text {th }}$ traits, and $\$ \mathrm{~d} \$$ is the regression coefficient.

Due to their computational simplicity, and effective implementation on convolution images, we utilize SVR. The main object of the SVR problem is to find the hyper plane which is close to majority pieces of the training landmarks as potential [16]. Assume that $N$ training points $\left(x_{1}, y_{1}\right),\left(x_{2}, y_{2}\right), \ldots,\left(x_{N}, y_{N}\right)$ with $x_{i}$ belong to $R$ and $y_{i}$ belong to $R, i=\overline{1, n}$. In this case, we must construct a specific hyper planes and qualities contains the information taken from $w$ and $b$. The hyperplane $w$ might be chosen to a low standard when minimizing aggregate distances from hyperplane, w, training points. Essentially, Utilize Vapnik's $\pi$ insensitive loss function as:

$$
\left|y_{i}-\left(w x_{i}+b\right)\right|_{\epsilon}=\left\{\begin{array}{c}
0:\left|y_{i}-\left(w x_{i}+b\right)\right| \leq \epsilon \\
\left|y_{i}-\left(w x_{i}+b\right)\right|-\epsilon: \text { Otherwise }
\end{array}\right.
$$

Three patch expert response maps: (A) face contour, (B) nose ridge, and (C) chin part. Logistic response maps of regressors that use strength contain strong responses around the edges, making it difficult to locate the actual location of the element. Our solution mitigates the aperture issue by incorporating response maps from both the strength and depth images.

The user may choose the value of $\varepsilon$, and the regularization $c$ controls the trade-off between hyperplane discovery with a massive regression execution. The QP problem associated with SVR can be expressed as:

$$
\min _{w, b, \xi, \xi^{*}} \frac{1}{2} w^{t} w+c\left(\sum_{i=1}^{L} \xi_{i}+\sum_{i=1}^{L} \xi_{i}^{*}\right)
$$

Such that

$$
w^{t} \phi\left(x_{i}\right)+b-y_{i} \leq \epsilon+\xi_{i}
$$


Example pictures of pressure, depth, and combined response maps (Figure 2 shows the patch expert method tested around the pixels of an initial estimate); A major issue faced by CLM is the aperture dilemma, where confidence in identification around the edge is higher than along it, which in the case of strength response maps is especially obvious for the nose ridge and face outline. Adding the depth information helps solve this problem, as the strong edges in both images do not necessarily correlate, allowing further disambiguation of points around strong edges.

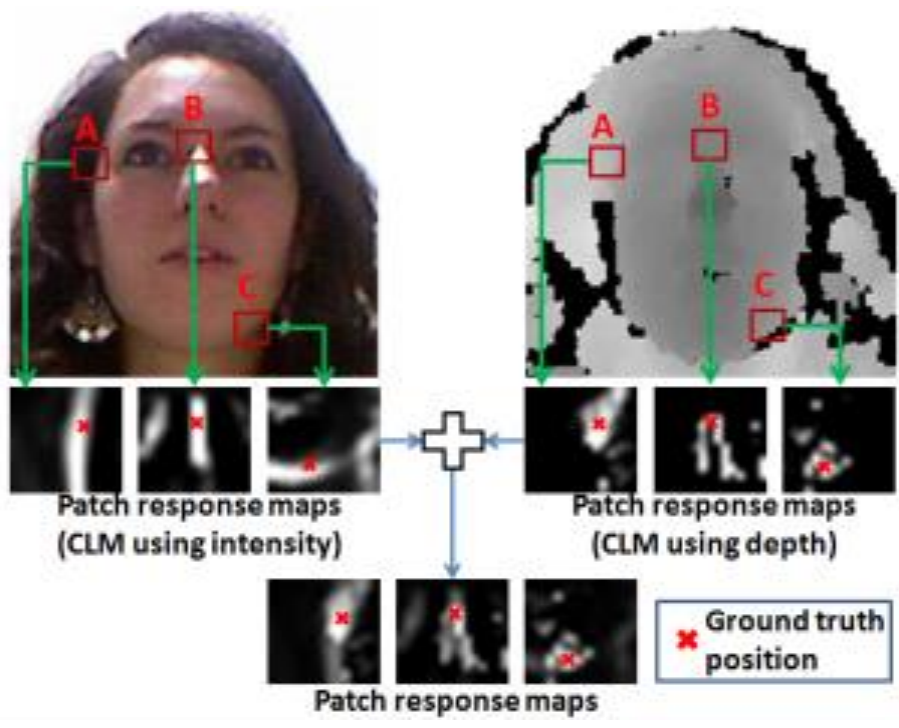

Figure 2. Three patch expert's response maps

\subsection{Fitting}

We use a standard two-step CLM fitting technique [6], [17], [18] perform a thorough local search around the current feature point estimation leading to a response map around each feature point, and then iteratively update the model parameters to optimize (2) until a convergence metric is reached. For fitting we utilize non-uniform regularized landmark mean shift (NU-RLMS) [2]. Provided for a starting CLM parameter evaluates $p$, NU-RLMS iteratively finds an overhaul parameter $P$, We assume that the non-rigid shape parameters $q$ differ by Gussian distribution with the variance of the $i^{t h}$ parameter corresponding to the non-rigid deformation mode's own value; the rigid parameters $s, R$, and $t$ obey a non-informative uniform distribution. Treating the positions of the actual landmarks as unknown variables will marginalize them out of the probability of aligning the landmarks:

$$
p\left(l_{i} \mid x_{i}, I, Z\right)=\sum_{y_{i} \in \psi_{i}} p\left(l_{i} \mid x_{i}, I, Z\right) p\left(y_{i} \mid x_{i}\right)
$$

Where $p\left(y_{i} \mid x_{i}\right)=N\left(y_{i}, x_{i}\right), p I$, with $p$ signify the contrast of noise in landmark locations resulting from PCA truncation in PDM construction, and $\psi_{i}$ signify all integer locations within the patch area. By substituting (8) into (2) we get:

$$
p(P)=\prod_{i=1}^{n} \sum_{y_{i} \in \psi_{i}} p\left(l_{i} \mid x_{i}, I, Z\right) N\left(y_{i} ; x_{i}, p I\right)
$$

The MAP term in (9) can be maximized using expectation maximization. The discrepancy between the available implementation and the original algorithm is attributed, in addition to frontal, to the use of qualified patches using profile face pictures. This leads to three sets of classifiers (frontal, left, right), with no answer functions for the occluded landmarks for the left and right sets. This allows us to deal with self occlusion since the invisible points for the fitting procedure are not evaluated.

\subsection{Face database}

In this paper, we used the labeled face parts in the wild (LFPW) database [15], a facial image database that includes precise information about the gender and age of each image. The LFPW image database was used to evaluate the proposed approach's efficiency and accuracy. The test group will consist of 700 face images, each of which has 68 landmarks. The number of lookup locations has a measurable effect on the position outcome. The proposed model's performance is compared to that of existing models. 


\section{DIFFERENT BETWEEN CLM AND A-CLM}

The below picture explains the different between original CLM and A-CLM. The above one is CLM and the below one is A-CLM. From Figure 3 one can see that the main different of CLM and A-CLM as show in CLM depends on one patch to extract the feature from the face whereas A-CLM depends on three patches to extract the feature from the face. The other different in fitting stage as shown in Figure 3.
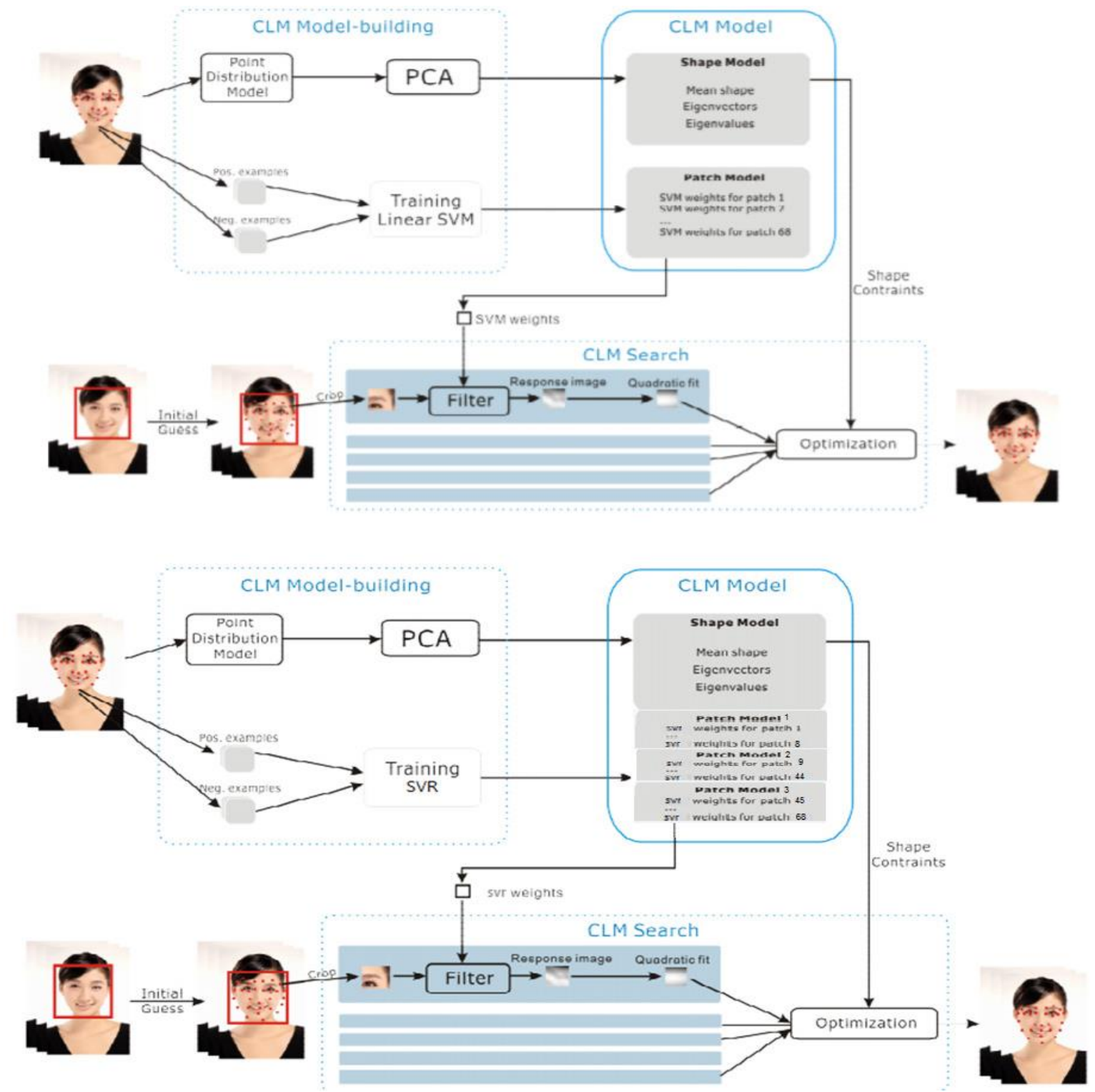

Figure 3. Difference between CLM and A-CLM

\section{Experimental results}

\subsection{CLM results}

This section details out the result of the CLM as facial feature extractors together with machine learning algorithms as the classifiers to estimate gender. The performance of each classifier was determined using two distinctive measurements: mean absolute error (MAE) and cumulative score (CS).

Table 1 shows the MAE of the machine learning classifiers i.e. SVM, SVR, KNN, and PT-SVM using CLM as the facial feature extraction models and SVR, CLNF as patch experts. To examine the performance of various machine learning algorithms as classifiers for gender estimation, experiments were conducted to test the classifier performance with CLM, using CLNF and SVR as patch experts separately, CLM as the feature extraction methods using LFPW databases. The machine learning algorithms or classifiers used in this study are 
PTSVM, SVM, SVR, and KNN. The results of this experiment are outlined in Figures 4 and 5. In the Table 2, we provide the efficiency of CLM with two different patch experts and four classifiers.

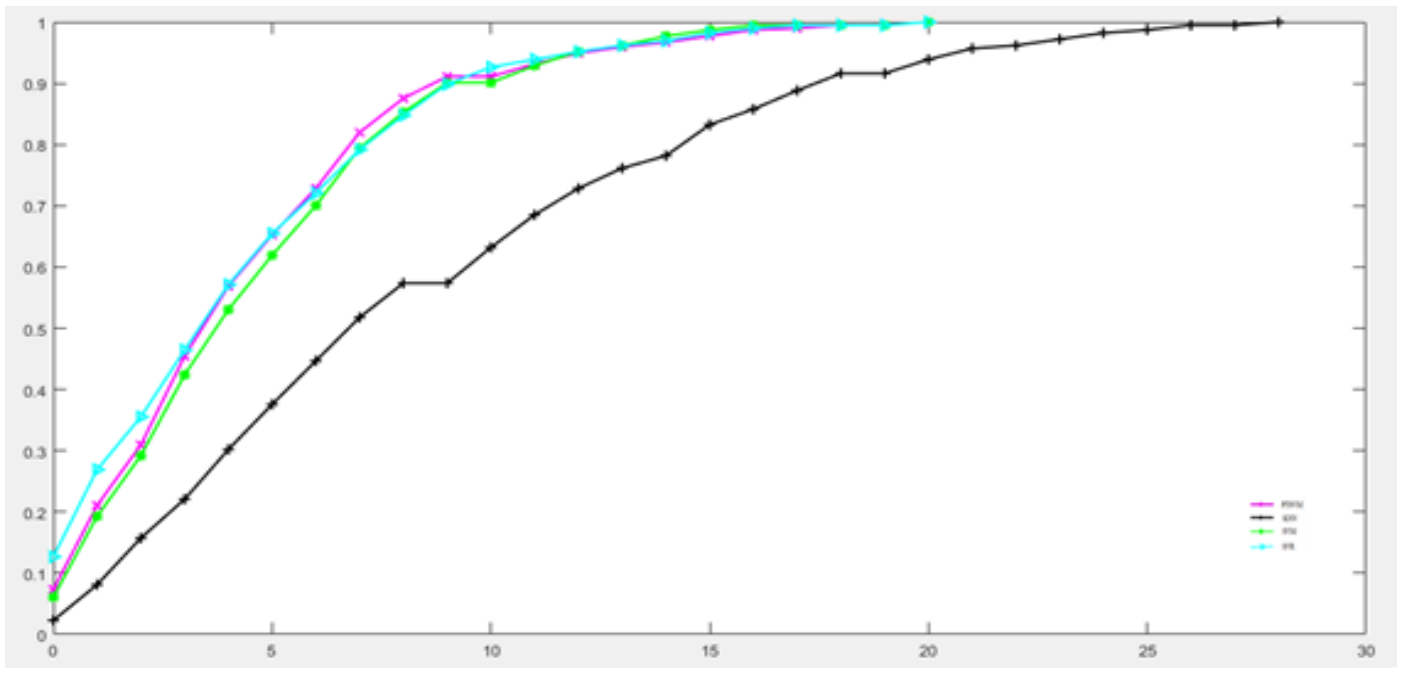

Figure 4. Cumulative score curves of the gender prediction methods using CLM with CLNF

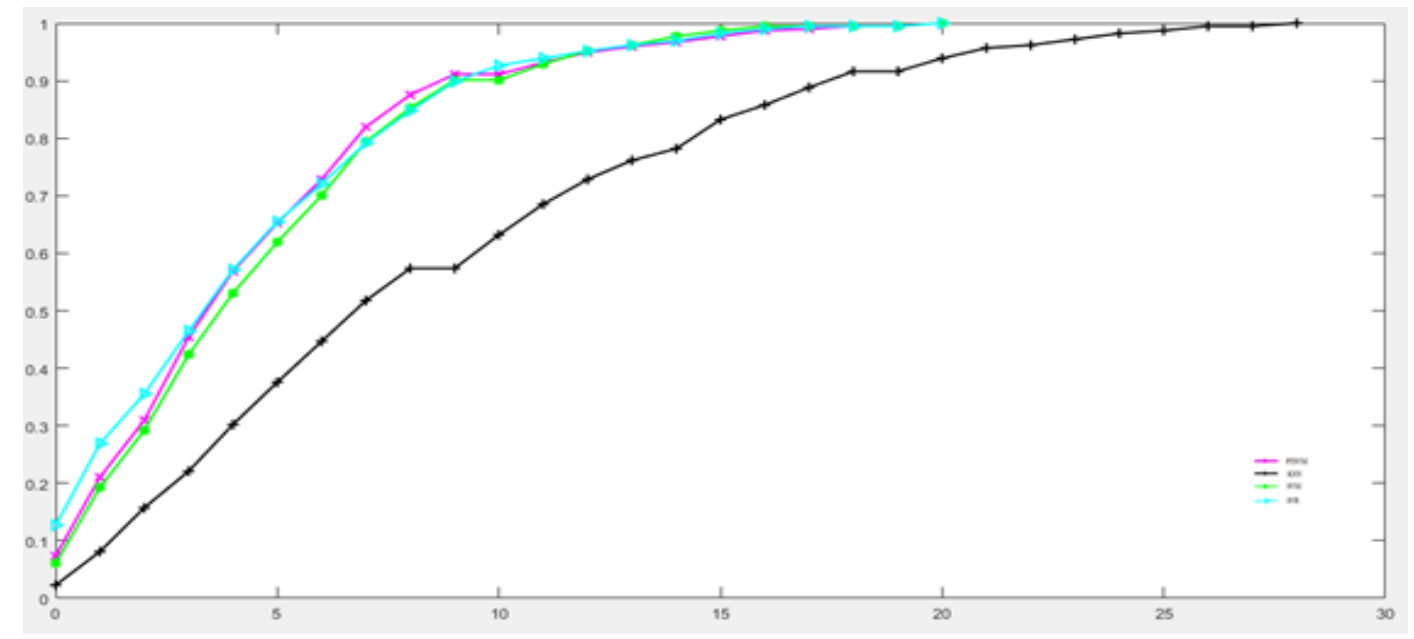

Figure 5. Cumulative score curves of the gender prediction methods using CLM with SVR

Table 1. MAE of the gender estimation techniques

\begin{tabular}{ccccc}
\multicolumn{5}{c}{ using CLM } \\
\hline Method & SVM & SVR & KNN & PT-SVM \\
\hline SVR & 4.25 & 4.67 & 9.58 & 4.24 \\
CLNF & 4.91 & 4.97 & 8.54 & 4.74 \\
\hline
\end{tabular}

Table 2. Efficiency of the gender prediction

\begin{tabular}{ccccc}
\multicolumn{4}{c}{ algorithms using CLM } \\
\hline Method & SVM & SVR & KNN & PT-SVM \\
\hline SVR & 0.065847 & 0.079852 & 0.040545 & 0.076524 \\
CLNF & 0.075214 & 0.083695 & 0.095874 & 0.071585 \\
\hline
\end{tabular}

\subsection{A-CLM results}

This section presents the result of the proposed model A-CLM as the facial feature extractor together with various machine learning algorithms to estimate gender. The performance of each classifier was determined using two distinctive measurements: MAE and CS. Table 3 shows the MAE of all machine learning algorithms (SVM, SVR, KNN, PT-SVM) using the A-CLM for facial feature extraction. Figure 6 indicates the Cumulative Score of the proposed model as the feature extraction method with various machne learning algorithms (KNN, SVR, SVM, and PTSVM) as classifiers.

Table 4 gives the average processing time to estimate gender using the proposed model as the feature extraction method and images from the LFPW database. The average prediction time is the time taken for the classifier to train and test the images. In Table 4, the result is given with respect to the machine learning algorithms (PTSVM, SVM, SVR, and KNN). 


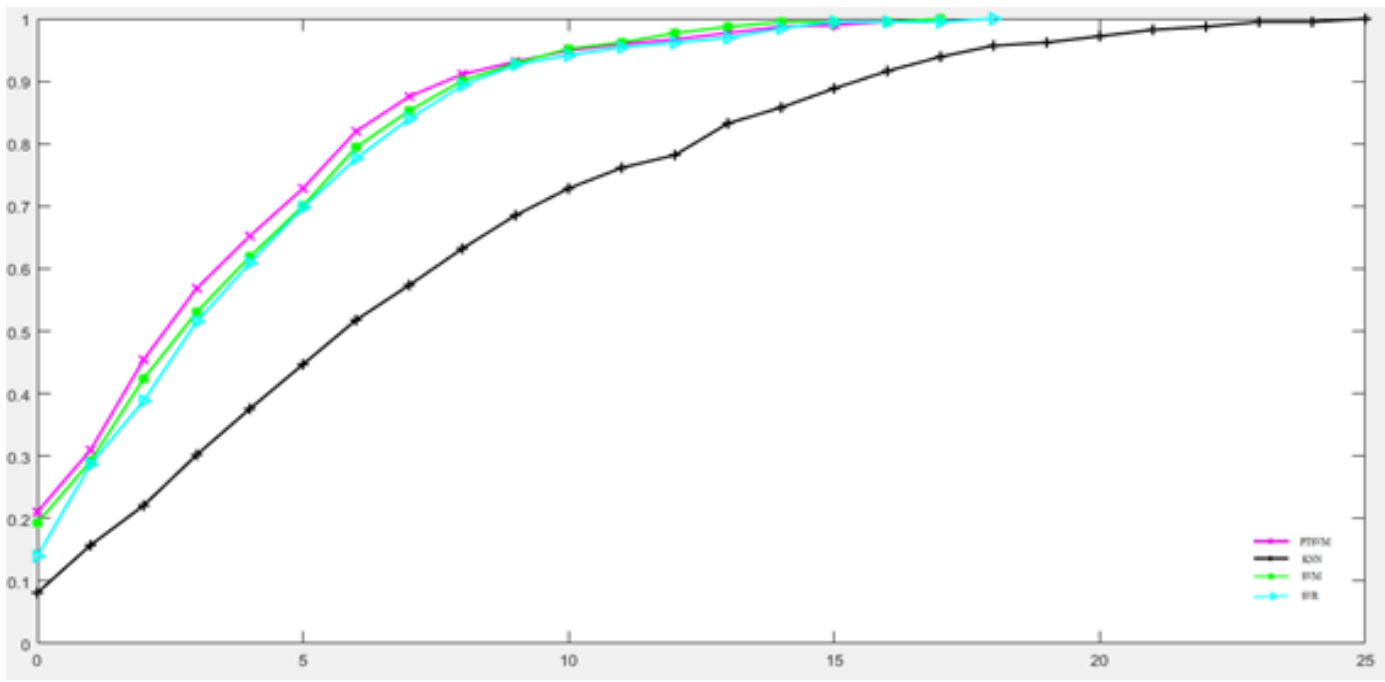

Figure 6. Cumulative score curves of the gender prediction methods using A-CLM

Table 3. MAE of the gender prediction algorithms

\begin{tabular}{ccccc}
\multicolumn{5}{c}{ using A-CLM } \\
\hline Method & SVM & SVR & KNN & PT-SVM \\
\hline A-CLM & 4.44 & 4.38 & 8.14 & 3.64
\end{tabular}

Table 4. Efficiency of the gender prediction algorithms using A-CLM

\begin{tabular}{ccccc}
\hline Method & SVM & SVR & KNN & PT-SVM \\
\hline A-CLM & 0.059845 & 0.078369 & 0.055145 & 0.079852
\end{tabular}

\section{CONCLUSION}

The implementation of the proposed model and the findings obtained from it were discussed in this study. The experimental results for estimating face gender were reviewed in detail. In this study, the effectiveness of the suggested model using various classifiers was evaluated. The experimental results demonstrate that our suggested model for gender prediction using PT-SVM provides more accurate results when compared to other classifiers, as shown by our results. As a bonus, our proposed model provides more accurate results when compared to the original CLM.

\section{ACKNOWLEDGEMENTS}

This work is funded by the Faculty of Informatics and Computing, Universiti Sultan Zainal Abdin (UniSZA), Terengganu, Malaysia.

\section{REFERENCES}

[1] L. Chen, C. Fan, H. Yang, S. Hu, L. Zou, and D. Deng, "Face age classification based on a deep hybrid model," Signal, Image and Video Processing, vol. 12, no. 8, pp. 1531-1539, 2018, doi: 10.1007/s11760-018-1309-6.

[2] A. Hassan and S. Viriri, "Invariant feature extraction for facial recognition: A survey of the state-of-the-art," 2018 Conference on Information Communications Technology and Society (ICTAS), 2018, pp. 1-6, doi: 10.1109/ICTAS.2018.8368754.

[3] B. Johnston and P. de Chazal, "A review of image-based automatic facial landmark identification techniques," EURASIP Journal on Image and Video Processing, vol. 2018, no. 1, p. 8, 2018, doi: 10.1186/s13640-018-0324-4.

[4] T. F. Cootes, "Active shape models-their training and application," Computer vision and image understanding, vol. 61, no. 1, pp. 38-59, 1995, doi: 10.1006/cviu.1995.1004.

[5] S. A. Adeshina and T. F. Cootes, "Automatic model matching using part-based model constrained active appearance models for skeletal maturity," 2015 Twelve International Conference on Electronics Computer and Computation (ICECCO), 2015, pp. 1-5, doi: 10.1109/ICECCO.2015.7416876.

[6] D. Cristinacce and T. F. Cootes, "Feature detection and tracking with constrained local models," in Procedings of the British Machine Vision Conference 2006, 2006, doi: 10.5244/C.20.95.

[7] M. Iqtait, F. S. Mohamad, and M. Mamat, "Feature extraction for face recognition via Active Shape Model (ASM) and Active Appearance Model (AAM)," IOP Conference Series Materials Science and Engineering, vol. 332, p. 012032, 2018, doi: 10.1088/1757-899X/332/1/012032.

[8] A. Zadeh, T. Baltrušaitis, and L.-P. Morency. "Deep constrained local models for facial landmark detection," arXiv preprint arXiv:1611.08657, vol. 3, no. 5. p. 6, 2016.

[9] S. Zahraddeen, S. Fatma and A. Abdulganiyu, "An efficient discrete cosine transforms and gabor filter-based feature extraction for face recognition," 6th International Conference on Postgraduate Education (ICPE), At: Universiti Teknikal, Melaka, Malaysia, 2014.

[10] X. Geng, Q. Wang and Y. Xia, "Facial Age Estimation by Adaptive Label Distribution Learning," 2014 22nd International Conference on Pattern Recognition, 2014, pp. 4465-4470, doi: 10.1109/ICPR.2014.764. 
[11] H. Wang, J. Hu and W. Deng, "Face Feature Extraction: A Complete Review," in IEEE Access, vol. 6, pp. 6001-6039, 2018, doi: 10.1109/ACCESS.2017.2784842.

[12] A. K. Sharma, U. Kumar, S. K. Gupta, U. Sharma, and S. L. Agrwal, "A Survey on Feature Extraction Technique for Facial Expression Recognition System," 2018 4th International Conference on Computing Communication and Automation (ICCCA), 2018, pp. 1-6, doi: 10.1109/CCAA.2018.8777550.

[13] W. Wei, Q. Jia, F. Yogli, G. Chen, and M. Chu, "Multi-modal facial expression feature based on deep-neural networks," Journal on Multimodal User Interfaces, vol. 14, no. 5, pp. 1-7, 2019, doi: 10.1007/s12193-019-00308-9.

[14] S. Tan, D. Chen, C. Guo, and Z. Huang, "A robust shape reconstruction method for facial feature point detection," Computational intelligence and neuroscience, vol. 2017, p. 4579398, 2017, doi: 10.1155/2017/4579398.

[15] C. Sagonas, "300 faces in-the-wild challenge: Database and results," Image and vision computing, vol. 47, pp. 3-18, 2016, doi: 10.1016/j.imavis.2016.01.002.

[16] A. J. Smola and B. Schölkopf, “A tutorial on support vector regression,” Statistics and Computing, vol. 14, no. 3, pp. 199-222, 2004, doi: 10.1023/B: STCO.0000035301.49549.88.

[17] L. Gu and T. Kanade, "A generative shape regularization model for robust face alignment," in Lecture Notes in Computer Science, Berlin, Heidelberg: Springer Berlin Heidelberg, 2008, pp. 413-426, doi: 10.1007/978-3-540-88682-2_32.

[18] J. M. Saragih, S. Lucey, and J. F. Cohn, "Deformable model fitting by regularized landmark mean-shift," International Journal of Computer Vision, vol. 91, no. 2, pp. 200-215, 2011, doi: 10.1007/s11263-010-0380-4.

[19] A. Zadeh, Y. C. Lim, T. Baltrušaitis and L. Morency, "Convolutional Experts Constrained Local Model for 3D Facial Landmark Detection," 2017 IEEE International Conference on Computer Vision Workshops (ICCVW), 2017, pp. 2519-2528, doi: 10.1109/ICCVW.2017.296.

[20] A. Asthana, S. Zafeiriou, S. Cheng and M. Pantic, "Robust Discriminative Response Map Fitting with Constrained Local Models," 2013 IEEE Conference on Computer Vision and Pattern Recognition, 2013, pp. 3444-3451, doi: 10.1109/CVPR.2013.442.

[21] Yang Wang, S. Lucey and J. F. Cohn, "Enforcing convexity for improved alignment with constrained local models," 2008 IEEE Conference on Computer Vision and Pattern Recognition, 2008, pp. 1-8, doi: 10.1109/CVPR.2008.4587808.

[22] G. Antipov, S.-A. Berrani and J.-L. Dugelay, "Minimalistic CNN-based ensemble model for gender prediction from face images," Pattern Recognition Letters, vol. 70, pp. 59-65, 2016, doi: 10.1016/j.patrec.2015.11.011

[23] K. Ito, H. Kawai, T. Okano and T. Aoki, "Age and Gender Prediction from Face Images Using Convolutional Neural Network," 2018 Asia-Pacific Signal and Information Processing Association Annual Summit and Conference (APSIPA ASC), 2018, pp. 7-11, doi: 10.23919/APSIPA.2018.8659655.

[24] I. Rafique, A. Hamid, S. Naseer, M. Asad, M. Awais and T. Yasir, "Age and Gender Prediction using Deep Convolutional Neural Networks," 2019 International Conference on Innovative Computing (ICIC), 2019, pp. 1-6, doi: 10.1109/ICIC48496.2019.8966704.

[25] A. Rattani, N. Reddy and R. Derakhshani, "Convolutional neural networks for gender prediction from smartphone-based ocular images," IET Biometrics, vol. 7, no. 5, pp. 423-430, 2018, doi: 10.1049/iet-bmt.2017.0171.

[26] M. R. Dileep and A. Danti, "Human age and gender prediction based on neural networks and three sigma control limits," Applied Artificial Intelligence, vol. 32, no. 3, pp. 281-292, 2018, doi: 10.1080/08839514.2018.1451217.

\section{BIOGRAPHIES OF AUTHORS}
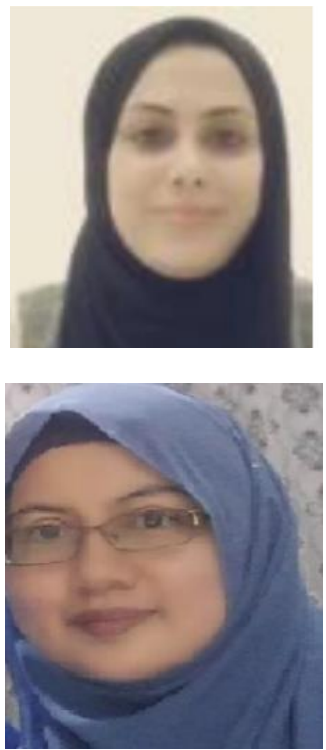

Ayah Alsarayreh (D) $\mathrm{SC}$ P is a Ph.D. candidate at the Faculty of Informatics and Computing, Universiti Sultan Zainal Abidin Malaysia, where she is now enrolled. Her research interests are in the subject of human biometrics, specifically in facial recognition. She can be contacted at email: aya_izzat_89@yahoo.com.
Fatma Susilawati Mohamad (iD) $\mathrm{SC} P$ is an Associate Professor in School of Information Technology, Faculty of Informatics and Computing, Universiti Sultan Zainal Abidin, Terengganu, Malaysia. She obtained her BSc in Information System from Oklahoma City University, USA in 1997. Then she pursued her master's degree in computer science from Universiti Kebangsaan Malaysia in 2004. In 2012, she obtained her PhD in Computer Science specializing in Pattern Recognition from Universiti Teknologi Malaysia. Currently, she is appointed as Deputy Director of Research Institute for Islamic Product and Malay Civilization. Dr Fatma is acted as Head of Image Processing and Pattern Recognition Research Group at the faculty. At present, she involved in research in Deep Learning apart of human biometric identification. Dr Fatma is an experienced lecturer. She has more than 20 years experiences in teaching and research. She has taught various courses both in undergraduate and postgraduate level over the past 20 years. Dr Fatma has published more than 100 journal articles, proceedings, and academic books to date. She has graduated more than $20 \mathrm{PhD}$ and Master students at present. Due to her vast experience and knowledge, Dr Fatma has been invited to deliver her keynote and invited speech at several conferences and academic seminars local or internationally. She is also being invited to give talk in academic and research for several institutions local and international. Besides, Dr Fatma also being appointed as an Academic Advisor and evaluator for postgraduate program local and internationally. She can be contacted at email: farie999@gmail.com. 